\title{
Integration of palliative care to the emergency department. Management algorithm approach
}

\author{
Eduardo D. Anica-Malagón ${ }^{1 *}$, Miguel Cordero-Pelayo ${ }^{2}$, Yareni N. Salgado-Abrego ${ }^{1}$, \\ Guillermo R. Vilchis-Palacios ${ }^{1}$, and Jorge A. Sosa-Bolio ${ }^{1}$ \\ ${ }^{1}$ Medical Emergency Service, Unidad 401, Hospital General de México "Dr. Eduardo Liceaga"; ${ }^{2}$ Service Anesthesiology, Centro Médico Dalinde. \\ Mexico City, Mexico
}

\begin{abstract}
Regarding medical emergencies, physicians exclusively deal with seriously ill patients suffering from advanced, progressive, and chronic diseases, where the diagnostic and therapeutic approach becomes simultaneous. Sometimes with a very wide range of possibilities that lead to a greater complexity when identifying if the patient is in the last phase of life. Thus, there is a risk of falling into therapeutic obstinacy or disinterest leaving aside the main objective of ensuring the comfort of these patients. In response to this need, our work team carried out an algorithm that aims to integrate palliative care to the emergency service, by identifying end-of-life patients, in addition to implementing management proposals for each stage thereof.
\end{abstract}

Key words: Palliative medicine. Emergency medicine. Palliative phase. Terminal patient. Dying patient.

\section{Introduction}

The incidence of chronic diseases has increased in recent years worldwide and due to advances in medical and surgical treatments; a longer survival time has been achieved for these patients with anatomical and functional repercussions. Often with significant deterioration in quality of life; in the attempt to maintain life, there is a risk of falling into therapeutic obstinacy by prioritizing time of life over quality of life ${ }^{1,2}$.

Faced with the great challenge of managing a patient with an advanced, progressive and incurable disease, the need for the creation of palliative care arose; Dr. Cicely Saunders was a pioneer in this area. Cicely Saunders developed the definition of total pain in 1964, in which she emphasized the importance of managing the physical, psychological, and spiritual pain of death as the preamble and the foundations of palliative care. Later in 1967, she managed to materialize this doctrine by establishing the first Hospice in St Christopher's, London, where only patients in the terminal phase were cared for, concentrating on achieving total pain relief until the patient's death ${ }^{3}$.

At present, there are several definitions of palliative care, the one with the greatest impact in the medical field is the one proposed by the World Health Organization, which defines it as: "an approach that improves the quality of life of patients (adults and children) and their relatives when they are facing problems inherent to a life-threatening illness. They prevent and alleviate suffering through early identification, assessment, and correct treatment of pain and other problems, whether physical, psychosocial, or spiritual"4.

\section{Correspondence:}

*Eduardo D. Anica-Malagón

E-mail: anicamalagon@yahoo.com
Date of reception: $31-08-2020$

Date of acceptance: 09-11-2020

DOI: $10.24875 /$ HGMX.20000079
Available online: 19-04-2021 Rev Med Hosp Gen Mex. 2021;84(2):87-91 www.hospitalgeneral.mx NC-ND license (http://creativecommons.org/licenses/by-nc-nd/4.0/). 
In the case of our country, Mexico, according to the current official standard NOM-011-SSA3-2014, criteria for the care of terminally ill patients through palliative care, defines it as "active and total care of those diseases that do not respond to curative treatment. The control of pain and other symptoms, as well as attention to psychological, social, and spiritual aspects"5.

It is of utmost importance to highlight that the population receiving end-of-life care is not a homogeneous population with unique characteristics; on the contrary, it is a progressive classification based on the evolution of the disease, which is why we can divide the final phase of the patient's life into the following stages: non-terminal, terminal, and agonal.

Non-terminal patients are those with advanced, progressive, and incurable disease. This also means a lack of reasonable possibilities of response to specific treatment, together with numerous problems and/or multiple, intense, multifactorial, and progressive symptoms with great emotional burden on the patient, family, and therapeutic team. It is closely related to the presence, explicit or not, of a limited life prognosis and a near death, but with a life expectancy of more than 6 months. When survival is $\leq 6$ months, then the patient is in the terminal phase ${ }^{3}$. When terminal patients present a sudden decrease in vital functions with an estimated survival time between 24 and $48 \mathrm{~h}$, they become by definition an agonal patient, mainly this group of patients should have a medical approach of providing comfort until death ${ }^{3}$.

Patients in the final phase of life may present exacerbations of symptoms according to their pathology, or the presence of concomitant potentially curable acute pathologies, and are therefore not exempt from requiring care in the emergency department.

The patient in the final phase of life has been integrated into a model of care, which allows us to understand the complexity thereof and establish the main guidelines in its management, ranging from the diagnosis of advanced, progressive, and incurable disease through the death of the patient and ending with the management of bereavement in the family $6-8$.

In medical training, preserving life is deep-rooted as a basic principle, because death is conceptualized as a defeat for physicians in the management of their patients and not as the last phase of life. For its part, emergency medicine is the specialty that, driven by acute symptoms, provides simultaneous diagnostic and therapeutic interventions in a timely manner to limit complications, as well as loss of function and life ${ }^{9,10}$. Emergency physicians are in a constant struggle to preserve life in the face of catastrophic scenarios, so it is not difficult to fall into therapeutic obstinacy. In addition to not having adequate training to recognize and manage the patient in the final phase of life in any of its stages, which can lead to stigmatizing the patient with advanced, progressive, and incurable disease as a patient who does not require interventions on their part, even when presenting a potentially curable acute disease ${ }^{11-13}$.

When a patient in the end-of-life phase requires care in the emergency department, emergency physicians should be able to decide which integration category should be used, according to the needs and characteristics of each particular patient. Considering that, patients could make use of the traditional consultation where they would request support from the palliative physician to carry out the immediate assessment and management. Alternatively, emergency physicians could team up with palliative care staff and work on the basis of previously established common goals; or advanced integration where they share the same physical space, goals, and protocols. Another option to consider is advanced integration where the EP would have the necessary skills, certified by a palliative care board to ensure appropriate training of the EP in the area of palliative medicine ${ }^{14-16}$. At present, none of these models have been established in Mexico or Latin America at any level of medical care. Neither were similar protocols found by means of a directed search in academic search engines such as PubMed using the MeSH terms "palliative care" and "emergency room;" nor with the Google scholar search engine using the same terms. This is perhaps due to the lack of knowledge of palliative care by emergency medical services, despite the fact that in some hospitals this service is already available. The relationship between both specialties is null or very difficult, because the objectives are different in both specialties and the emergency department can do little for the palliative patient even with a potentially curable acute pathology. Thus, its main objective is limited to preserving life, even falling into therapeutic obstinacy without visualizing other objectives in the short-, medium-, and long-term or reaching the opposite extreme by not considering it as a therapeutic challenge and falling into disinterest, poor quality of care, and therapeutic abandonment ${ }^{17,18}$.

Hence, our team, which has training in emergency medicine and palliative medicine, developed an algorithm to facilitate the care and management of patients in the final phase of life who come to the emergency department of our hospital unit. The complexity of 


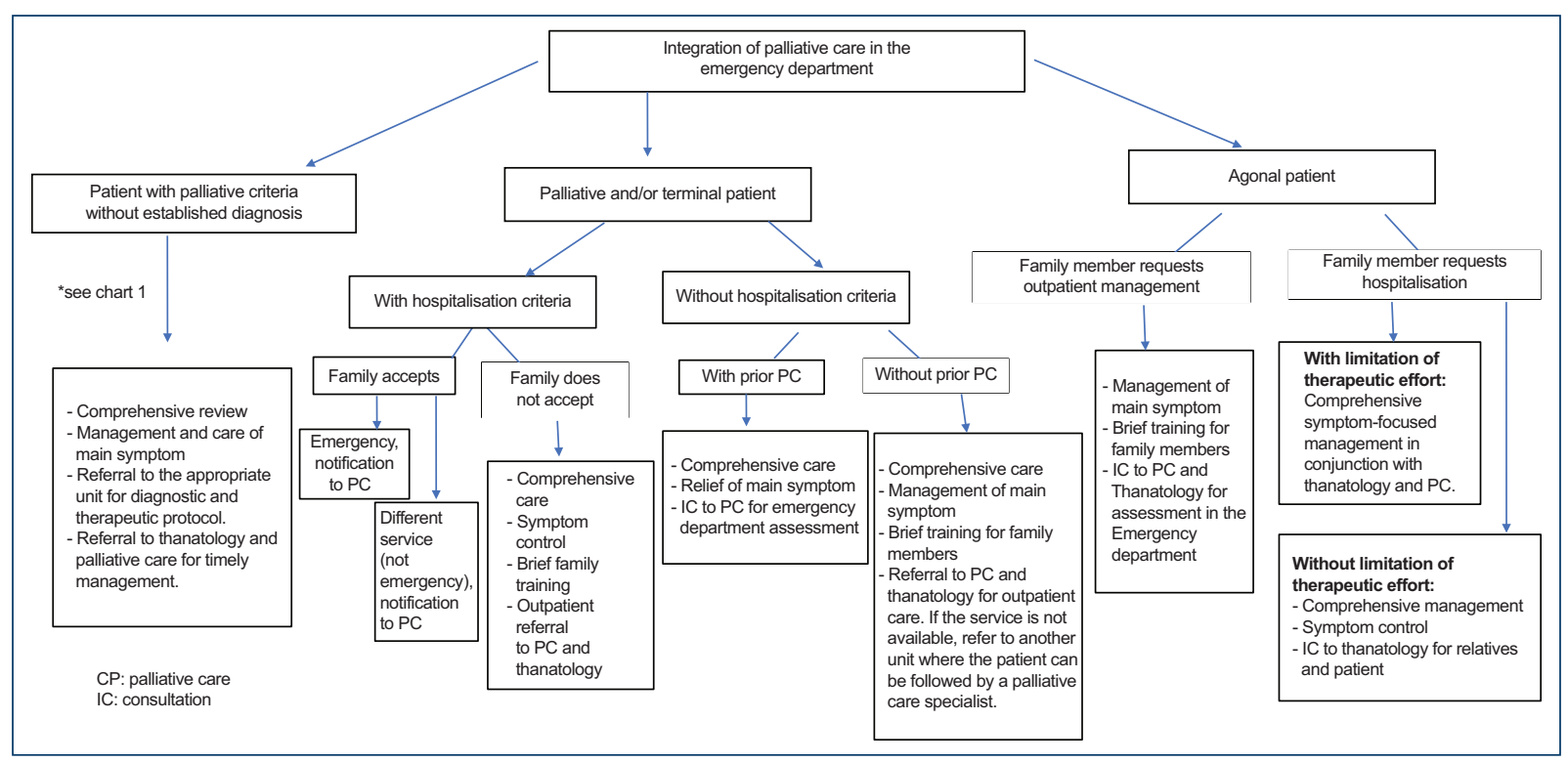

Figure 1. Algorithm for proposing the integration of palliative care into the emergency department.

these patients is taken into account, ranging from a patient with characteristics of advanced, progressive, and incurable disease without an established diagnosis, to the agonal patient. This includes cancer and non-cancer patients, without forgetting their particular characteristics (Fig. 1) ${ }^{19-21}$.

For the purposes of our management algorithm, the end-of-life patient seeking emergency medical care will be classified as follows:

1. Patient with diagnostic suspicion compatible with a pathology that cannot be reversed medically or surgically.

2. End-of-life patient: patient with the diagnostic certainty of suffering from an advanced, progressive, and incurable disease.

3. Patient with an advanced, progressive, and incurable disease without terminal criteria: patient with an advanced, progressive, and incurable disease with a life expectancy of more than 6 months.

4. Terminally ill patient: patient with an advanced, progressive, and incurable disease with an estimated survival due to underlying pathology of 6 months or less.

5. Patient in the agonal phase: terminal patient with a sudden decrease in vital functions with a prognosis of survival of no more than $48 \mathrm{~h}^{3}$.

Within the approach to palliative care patients in the $E D$, we decided to divide them into three groups: patients with criteria of advanced, progressive, and incurable disease but without an established diagnosis, those with a diagnosis of advanced, progressive, and incurable disease and/or terminal and agonal patients to simplify the approach.

It is evident that the main reason for rushing to an emergency department is the presence of a predominant and in some cases refractory symptom (pain, fatigue, dyspnea, and among the most relevant symptoms), so in our three groups this will be a common goal to achieve comfort regardless of the stage of the disease.

In the case of patients with an advanced, progressive and incurable disease in a non-terminal phase without an established diagnosis, their first right is to be sure of their diagnosis. Thus, we consider it appropriate to refer them to the corresponding area to carry out proper medical and/or surgical diagnostic and therapeutic protocol, because in our hospital, these services do not assess patients on an outpatient basis at the emergency department. The patient must go to these services, as well as to the thanatology and palliative care service for the psychosocial management of the pathology, thus addressing the patient in a comprehensive and appropriate manner to the pathology 22-25.

Regarding patients with advanced, progressive, and incurable illness with or without terminal criteria, a distinction should be made between those who benefit from hospitalization and those who do not, as well as whether this should be in an emergency department or whether they are in a position to move to a different department where the environment is nicer. It is worth mentioning that the family member should make this 
decision in agreement with the patient. If the patient does not accept hospitalization, family members will be the ones who should be trained to keep the patient in the best conditions, advised by the palliative medicine unit of our hospital through outpatient care, since this service does not have 24-h care or a hospitalization area. If the patient does not require hospital management, is already known by the palliative care staff and is within the opening hours of this service, a consultation with this service could be considered to treat the main symptom ${ }^{24}$.

Considering that patients in the agonal phase have an estimated life span of 24-48 h, the decision to hospitalize them becomes complicated, since it rarely offers greater comfort than could be had at home, knowing the prognosis, and outcome of the disease. In addition, many of these patients no longer have the capacity to make decisions, and because of the difficulty in dealing with end-of-life issues, many family members prefer that the patient die at the hospital. This was observed in a study of 5837 patients in palliative care programs with end-stage cancer that the patient was less likely to choose to die at home when the patient presented moderate or severe pain and when the patient presented an adequate functional state as measured by the Palliative Performance Scale. Moreover, it was identified that not all patients choose home, such as those who are not married, non-white patients, and the elderly ${ }^{26}$. When it comes to cardiopulmonary resuscitation, patients and their relatives often think that they have enough time to discuss this and other end-of-life issues. Hence, it is common that patients do not make decisions about this phase or do not communicate them with their relatives. Thus, it is common for them to reach the dying phase and be subjected to resuscitative measures that they may not have wanted. It has been identified that reaching the agonal phase without decision-making regarding resuscitation is associated with increased patient agony and poorer bereavement management by the family ${ }^{27}$.

\section{Conclusion}

The main task of ED physicians when faced with patients with an advanced, progressive, and chronic disease is to integrate the two medical disciplines in their management, understanding integration as the incorporation of palliative care into the ED consultation by correctly identifying this population, exploring the literature and advances in the area during their training, to put it into daily practice and identify the nearest centers that have a palliative care area if there is none in their unit, to finally have a plan at the end of the consultation as would be done with any other patient.

\section{Conflicts of interest}

The authors declare that they have any conflicts of interest.

\section{Ethical disclosures}

Protection of human and animal subjects. The authors declare that no experiments were performed on humans or animals for this study.

Confidentiality of data. The authors declare that no patient data appear in this article.

Right to privacy and informed consent. The authors declare that no patient data appear in this article.

\section{References}

1. Reuter Q, Marshall A, Zaidi H, Sista P, Powell ES, McCarthy DM, et al. Emergency department-based palliative interventions: a novel approach to palliative care in the emergency department. J Palliat Med. 2019; 22:649-55.

2. Kendall, V. Caring for patients at the end of life in intensive care units. End of Life Care. 2008:2:38-47. doi: 10.1136/EOLC-02-03.8.

3. Sociedad Española de Cuidados Paliativos, guía de Cuidados Paliativos; 2019. https://www.secpal.com/biblioteca_guia-cuidados-paliativos_2-definicion-de-enfermedad-terminal. [Last accessed on 2019 Oct ${ }^{15}$.

4. Fortalecimiento de los Cuidados Paliativos Como Parte del Tratamiento Integral a lo Largo de la vida. Resolución WHA; 67a Asamblea Mundial de la Salud; 2014.

5. NORMA Oficial Mexicana NOM-011-SSA3-2014, Criterios Para la Atención de Enfermos en Situación Terminal a Través de Cuidados Paliativos; 2014.

6. Lamba S, DeSandre PL, Todd KH, Bryant EN. Integration of palliative care into emergency medicine: the improving palliative care in emergency medicine (ipal-em) collaboration. J Emerg Med. 2014:46(2):264-70.

7. Green E, Ward S, Brierley W, Riley B, Sattar H, Harris T. They shouldn't be coming to the ED, should they? a descriptive service evaluation of why patients with palliative care needs present to the emergency department. Am J Hosp Palliat Med. 2017;34(10):984-90.

8. Henson L, Gao W, Higginson I, Smith M, Davies J, Ellis-Smith C, et al. Emergency department attendance by patients with cancer in the last month of life: a systematic review and meta-analysis. J Clin Oncol. 2015; 33:370-6.

9. Myburgh J, Abillama F, Chiumello D, Dobb G, Jacobe S, Kleinpell R. End-of-life care in the intensive care unit: report from the task force of world federation of societies of intensive and critical care medicine. J Crit Care. 2016;34:125-30.

10. Davies J, Vora V. Challenges of providing palliative care in a critical care setting. BMJ Support Palliat Care. 2014;4:A1-A110.

11. Aslakson R, Cheng J, Vollenweider D, Galusca D, Smith TJ, Pronovost PJ. Evidence-based palliative care in the intensive care unit: a systematic review of interventions. J Palliat Med. 2014;17:219-35.

12. Song MK, Happ MB. Generating high quality evidence in palliative and end-of-life care. Heart Lung. 2017;46(1):1-2.

13. Song MK, Happ MB. Generating high quality evidence in palliative and end-of-life care. Heart Lung. 2017:46:1-2.

14. Kraus CK, Greenberg MR, Ray DE, Morss S. Palliative care education in emergency medicine residency training: a survey of program directors, associate program directors, and assistant program directors. J Pain Symptom Manage. 2016;51:898-906.

15. Iyer AS. Early palliative care in advanced illness do right by mama. JAMA Intern Med. 2017;177:761-2.

16. Shoenberger J, Lamba S, Goett R, DeSandre P, Aberger K, Bigelow S, et al. Development of hospice and palliative medicine knowledge and skills for emergency medicine residents: using the accreditation council for graduate medical education milestone framework. AEM Educ Train. 2018:2:130-45. 
17. Nelson JE, Campbell ML, Cortez TB, Curtis JR, Frontera JA, Gabriel M, et al. Implementing ICU Screening Criteria for Unmet Palliative Care Needs: a Guide for ICU and Palliative Care Staff A Technical Assistance Monograph from the IPAL-ICU Project. 2013 The IPAL-ICU Project, Center to Advance Palliative Care, No. (ANCP76_66; 2013.

18. Byock I. Improving palliative care in intensive care units: identifying strategies and interventions that work. Crit Care Med. 2006:34:S302-5.

19. Boyle D, Barbour S, Anderson W, Noort J, Grywalski M, Myer J, et al. Palliative care communication in the ICU: implications for an oncology-critical care nursing partnership. Semin Oncol Nurs. 2017;33:544-54.

20. Connolly C, Miskolci1 O, Phelan D, Buggy DJ. End-of-life in the ICU: moving from "withdrawal of care" to a palliative care, patient-centred approach. Br J Anaesth. 2016;117:143-5.

21. Hurd C, Curtis JR. Education about palliative care in the intensive care unit: rediscovering opportunity. J Grad Med Educ. 2014;6:167-9.

22. Sihra L, Harris M, O'Reardon C. Using the improving palliative care in the intensive care unit (IPAL-ICU) project to promote palliative care consultation. J Pain Symtomp Manag 2011;42:672-5.
23. Knaul FM, Farmer PE, Krakauer EL. Alleviating the access abyss in palliative care and pain relief an imperative of universal health coverage: the lancet commission report. Lancet. 2018;391:1391-454.

24. Green E, Ward S, Brierley W, Riley B, Sattar H, Harris T. They shouldn't be coming to the ED, should they? a descriptive service evaluation of why patients with palliative care needs present to the emergency department. Am J Hosp Palliat Med. 2016;1-7.

25. Counselman FL, Babu K, Edens MA, Gorgas DL, Hobgood C, Marco CA, et al. The 2016 model of the clínica practice of emergency medicine. J Emerg Med. 2017;52:846-9.

26. Hui D, Morgado M, Chisholm G, Withers L, Nguyen Q, Finch C, et al. High-flow oxygen and bilevel positive airway pressure for persistent dyspnea in patients with advanced cancer: a phase II randomized trial. J Pain Symptom Manage. 2013;46:463-73.

27. Miyashita M, Morita T, Sato K, Hirai K, Shima Y, Uchitomi Y. Factors contributing to evaluation of a good death from the bereaved family member's perspective. Psychooncology 2008;17:612-20. 